\title{
Conference Paper \\ Integrating Greenhouse Gas Emissions Costs in Lifecycle Loss Evaluations: A Case Study for Transmission Lines
}

\author{
Antonis L. Lazari and Charalambos A. Charalambous \\ Department of Electrical and Computer Engineering, Faculty of Engineering, University of Cyprus, P.O. Box 20537, \\ 1687 Nicosia, Cyprus \\ Correspondence should be addressed to Antonis L. Lazari; lazari.antonis@ucy.ac.cy
}

Received 4 January 2013; Accepted 14 March 2013

Academic Editors: Y. Al-Assaf, P. Demokritou, A. Poullikkas, and C. Sourkounis

This Conference Paper is based on a presentation given by Antonis L. Lazari at "Power Options for the Eastern Mediterranean Region” held from 19 November 2012 to 21 November 2012 in Limassol, Cyprus.

Copyright ( 2013 A. L. Lazari and C. A. Charalambous. This is an open access article distributed under the Creative Commons Attribution License, which permits unrestricted use, distribution, and reproduction in any medium, provided the original work is properly cited.

Considering the investment volume in the electrical energy infrastructure and the increasing awareness for global warming and climate change, this paper aims to deliver an enhanced transmission line losses evaluation method that integrates true environment financial figures. The enormous volume of transmission lines utilized in power systems across the world provides a considerable potential for energy savings by adopting the idea of "energy-efficient transmission lines." In this paper the total owning cost (TOC) formula with built-in environmental components is used to assess the relative economic benefit of a high-first-cost, low-loss transmission line unit versus one with a lower first cost and higher losses. The proposed methodology is applied on the Cyprus Power System, by incorporating true financial data and system characteristics.

\section{Introduction}

European Commission strategies are encouraging communities towards more efficient and more sustainable energy solutions that will result in reduction of greenhouse gases (GHG) emissions in future years. Nowadays the reduction of greenhouse gas emissions is becoming a topical issue due to the growing concern for global warming and climate change. The actions that can immediately reduce GHG emissions and their cost, for an electric utility, are the use of energy-efficient equipment and renewable energy sources.

Recent studies have revealed that transmission lines have a key contribution to the total losses of a utility's system [1]. An "energy-efficient" transmission line may be used to lower electrical power generation and subsequent GHG emissions.

Loss evaluations endeavours are mainly utilized to inform a user regarding the optimum time to retire or replace existing transmission line units and other utility equipment. They are also widely employed to determine the relative economic benefit of a high-first-cost, low-loss unit versus one with a lower first cost and higher losses.
Furthermore, the emissions trading system (ETS) is a market-based approach used to control pollution by providing economic incentives for achieving reductions in the emissions of pollutants [2]. Thus, the economic cost of losses to a utility is directly affected by the incentives set by ETS. As a consequence, the factors that represent the emission reduction incentives need to be integrated in the conventional loss evaluation techniques [3-6]. Identical to energy prices, the GHG emissions are also assigned a price by energy markets that is the environmental cost (EC). The EC for a utility arises from the cost to buy emission credit allowances.

This paper aims to integrate the GHG emissions costs into the loss evaluation method of transmission lines. An extensive explanation of the new concepts is provided. In particular the method presented in this paper is used for the economic evaluation of transmission lines embedded in the Cyprus Power System. It should be noted that the results are benchmarked against the conventional loss evaluation principles [3]. True financial data and system characteristics are utilized in this paper. 


\section{Related Literature}

A detailed literature research has been performed to reveal the actual need for carbon emissions decrease and their influence in future losses evaluation endeavors.

2.1. Loss Evaluations Techniques and Models. In terms of loss evaluation techniques, it appears that most of the published literature refers to the loss evaluation of distribution transformers. The most complete methodology is found in two papers published in $1981[4,5]$. The first part refers to the total annual cost technique enhanced to account for energy cost inflation, load growth, and transformer change-out load. The second part presents the cost and load parameters used in the enhanced loss evaluation endeavor and a comparison review over other methods.

In 1991, the IEEE C57.120 [3] was formed to provide a universal method for establishing loss evaluation factors for power transformers and reactors. The standard methodology is based on the present worth of annual requirements which is equivalent to the total levelized annual cost method.

Furthermore, a paper which models the effect of a loss decrease in a power plant during an evaluation period was published in 1988 [6]. The suggested model was applied to investigate the effect of the loss evaluation technique in selecting the most efficient transmission line conductor.

Finally, the concept of integrating an environmental cost factor into the conventional loss evaluation technique was introduced in [7]. The methodology is based on the IEEE C57.120 loss evaluation method that further adopts an environmental cost factor for the evaluation of distribution transformer losses. The concept of a "reference transformer" for benchmarking the losses is thereby introduced.

\subsection{The Emissions Trading Scheme and the Kyoto Protocol.} Scientists and politicians have recognized the need for immediate actions against pollution, so as to protect the environment and, in general, the economy of their nations. For this reason, the United Nations Framework Convention on Climate Change has set in 1992 the practicalities of what is known today as the "Kyoto protocol" [2]. The principal aim of the protocol is the substantial reduction of GHG emissions over the next few years. This goal is implemented in phases and is subject to systematic review and development.

A descriptive analysis of the Kyoto protocol and its goals can be found in $[2,8]$. The existing GHG gases emissions trading systems and their foundations were analyzed indepth in $[9,10]$. The extensive literature review, also, provided information concerning GHG emission allowances, future predictions concerning the environmental factors, and the enforcements that could be done to achieve the long-term target.

It should be noted that the protocol mechanisms have initialized a trading scheme for human-related emission allowances or permits $\left(\mathrm{CO}_{2}\right.$, etc. $)$ in financial markets. What was set is a market-based approach to control pollution by providing economic motivations for achieving a decrease in the emissions of the pollutants. The main participants of the system are mainly the polluting industries, electric utilities, and the so-called "carbon" investors. The three schemes that have been set into act are (a) the emissions trading, (b) joint Implementation (JI) and (c) clean Development Mechanism (CDM) $[2,10]$.

In this case study the environmental cost factor (introduced in loss evaluations) is associated with the European Union-Emission Trading System (EU-ETS). The EU-ETS is the world's largest single market for GHG emission allowances (98\% of the world in 2007). The EU-ETS is a powerful tool that prevents the climate change in terms of issuing emission permits and prices. It is claimed to be the most effective way for countries to move towards a lowcarbon economy [9].

The provisions of the EU-ETS regulate, for each polluting industry, the right to emit a certain annual volume of GHG emissions into the atmosphere. The permits are issued as tradable assets with their price being set by the supply and demand of allowances at that time. One European Union Allowance (EUA) is the right to emit one tonne of $\mathrm{CO}_{2}$ in a year. The polluting industries can, in fact, "consume" their EUAs, that is, emit them, or decrease their emissions by reducing production or by using more technologically efficient methods. In any case, surplus EUAs can be sold in the market to those who emit more than allowed. In this way, the buyer of EUAs pays a fine for emitting more and the seller is rewarded for emitting less.

The benefits of the protocol over the future years largely outweigh the economic investments made for a lower-carbon world. The goal is technically feasible and economically affordable; GHG emissions to be stabilized by 2020 and to be reduced to at least $50 \%$ of 1990 levels by 2050 [2].

\section{Classical Loss Evaluation Method}

The loss evaluation techniques are utilized by electric utilities to provide information regarding transmission lines total cost over their entire lifetime-total owning cost (TOC). In addition to this, they are widely employed to determine the relative economic benefit of a high-first-cost, low-loss unit versus one with a lower first cost and higher losses. The most cost-effective and "energy-efficient transmission lines" are said to be those with the lowest TOC over an evaluation period (e.g., expected lifetime) [3-6]. More precisely, the TOC of a transmission line is the sum of its purchase price (PP) and its total cost of losses throughout its lifetime (COL) as given by (1).

$$
\mathrm{TOC}=\mathrm{PP}+\mathrm{COL}
$$

The total losses in transmission lines are the sum of line's no-load losses (NLL-kW) and load losses (LL-kW). Both NLL and LL are calculated on the basis of their demand $(€ / \mathrm{kW})$ and energy $(€ / \mathrm{kWh})$ components. The demand component is the cost of installing system capacity in $€ / \mathrm{kW}$ to serve the power used by the losses. The energy component is the present value of the energy that will be used by one kilowatt of loss during the life-cycle of the unit under study 
in $€ / \mathrm{kWh}$. Both demand and energy components are appropriately annuitized (i.e., levelized) to provide a total eurosper-kilowatt $(€ / \mathrm{kW})$ figure which accounts for the sum of the present worth of each kilowatt of loss of units throughout the transmission line life, or some other selected evaluation period. This figure represents the maximum amount that can be spent by a user on more efficient units to save a kilowatt of loss.

The total losses can be seen as a load to the system, thus more energy is required to meet the total demand (supply + losses). The transmission lines' load losses are those observed when the copper conductor is energized and the line is feeding power. The no-load losses in transmission lines are, in principle, due to the "corona effect." The effect of the "corona" losses is insignificant in comparison to load losses and thus no-load losses will be totally omitted in this study.

The COL term of (1) is expanded giving (2), keeping in mind that the no-load losses term is negligible in our study.

$$
\mathrm{COL}=B \times \mathrm{LL}
$$

Equation (2) expresses the cost of transmission line load losses throughout its lifetime. $B$ is the load loss cost factor $(€ / \mathrm{kW})$ and LL are the load losses $(\mathrm{kW})$. The load loss cost factor is computed according to [3]

$$
B=\frac{\mathrm{LIC} \times \mathrm{PRF}^{2} \times \mathrm{PUL}^{2}+\mathrm{LECL} \times \mathrm{TLF}^{2}}{\mathrm{ET} \times \mathrm{FCR} \times \mathrm{IF}},
$$

where LIC is the levelized annual transmission system investment cost $(€ / \mathrm{kW}-\mathrm{yr})$, LECL is the levelised annual energy and operating cost of load losses $(€ / \mathrm{kW}-\mathrm{yr}), \mathrm{PRF}$ is the peak responsibility factor that derives from the transmission line load at the time of the power system peak load divided by the line's peak load, PUL is the peak per-unit transmission line load that derives from the average of the annual peaks throughout the transmission line lifetime divided by the line rated load loss, ET is the efficiency of transmission, FCR is the fixed charge rate that represents the "cost of ownership" and IF is the increase factor that represents the total amount a user has to pay to acquire the transmission line, including the purchase price, overhead, fee, tax, and [3].

The levelised cost LECL is computed as in (4).

$$
\begin{aligned}
\mathrm{LECL}= & \mathrm{CRF} \times \mathrm{HPY} \times \mathrm{CYEC} \times\left(\frac{1+\mathrm{EIR}}{d-\mathrm{EIR}}\right) \\
& \times\left[1-\left(\frac{1+\mathrm{EIR}}{1+d}\right)^{\mathrm{BL}}\right]
\end{aligned}
$$

where CRF is the capital recovery factor that is computed by (5), HPY are hours of transmission lines operation per year (8760 hours), CYEC is the current year energy cost $(€ / \mathrm{kWh})$, EIR is the annual escalation rate of energy cost (\%), BL is the transmission line lifetime (years), and $d$ refers to the annual discount rate (\%). Consider

$$
\mathrm{CRF}=\frac{d \times(1+d)^{\mathrm{BL}}}{(1+d)^{\mathrm{BL}}-1}
$$

The peak per-unit load is calculated from

$$
\mathrm{PUL}=\frac{\mathrm{ITL}_{\mathrm{TPL}} \times(1+\mathrm{TPLIF})\left[(1+\mathrm{TPLIF})^{\mathrm{BL}}-1\right]}{\mathrm{BL} \times \mathrm{TPLIF}},
$$

where $\mathrm{ITL}_{\mathrm{TPL}}$ is the initial year transmission line load over the peak load and TPLIF is the peak load annual incremental factor (\%). The transmission line loading factor is derived from

$$
\mathrm{TLF}=\sqrt{\mathrm{LF} \times \mathrm{PUL}^{2}},
$$

where LF refers to the load loss factor $[3,4]$.

\section{Loss Evaluation Method with Environmental Cost}

As mentioned before, the target of this study is to redefine the conventional TOC to properly incorporate an environmental cost factor as presented in [7] for distribution transformers. The environmental cost in the losses evaluation endeavors is the financial cost to buy GHG emission credit allowances that are used to meet the transmission line losses [11].

The environmental cost (EC) is introduced in the conventional TOC (1) formula as per $(8)[7,12,13]$.

$$
\mathrm{TOCE}=\mathrm{TOC}+\mathrm{EC}
$$

The TOCE (8) completes the conventional loss evaluation method by incorporating the proposed environmental cost $(\mathrm{EC}-€)$.

In the context of environment protection, the Kyoto protocol foundations and the EU-ETS have set up an emission trading scheme in financial markets. Electric utilities are set under a GHG emission limit every year. In case of excess emissions that violate the limits, the utilities can either pay penalties or they can trade emission credits from other utilities [2]. This means that each utility handles its credits as a tradable asset and can "use" the credits in any way that is more financially efficient for the business.

As one can understand, the environmental cost is introduced in cases where a utility violates its emission allowances. In that case, more emission rights need to be traded (buy credits) by the polluting business, thus paying a fine for excess emissions. On the other hand, when the utility is emitting less than its yearly allowance, it has the right to sell its excess credits to businesses emitting more, thus being rewarded.

4.1. Reference Transmission Line. Each utility needs to assess its environmental emission allowances so as not to pay an excess emission penalty than already allowed. More specifically, electric utilities need to set the specifications of the so-called "reference transmission line", for each power rating. The specifications include the reference no-load losses $\left(\mathrm{NLL}_{r}-\mathrm{kW}\right)$ and reference load losses $\left(\mathrm{LL}_{r}-\mathrm{kW}\right)$. The reference unit losses are changed according to the operating conditions and the voltage rating of the system [11]. The specification of the reference figures is according to the contribution of the transmission line energy losses to the total 
GHG emissions of the utility under study. The transmission line's share in the violation of the maximum values imposed by international standards can also affect the decision for the reference unit. The reference figures must correspond to the maximum allowed losses per voltage rating that does not violate the GHG emission limit allocated to transmission lines.

For example, in the case study that follows, a reference load loss figure is used $(800 \mathrm{~kW})$ since the comparison made is between conductors having identical voltage level operation. The reference $800 \mathrm{~kW}$ losses represent the limit over which an environmental cost will be added on the loss evaluation method.

The environmental cost is computed by calculating the energy losses that remain from the difference of the total energy losses of the evaluated transmission line and the total energy losses of the reference transmission line (e.g., $800 \mathrm{~kW}$ ). The difference for the no-load losses is given by (9) and the difference for the load losses is given by (10). Consider

$$
\begin{gathered}
\Delta P_{\mathrm{NLL}}=P_{\mathrm{NLL}}-P_{\mathrm{NLL}_{r}}, \\
\Delta P_{\mathrm{LL}}=P_{\mathrm{LL}}-P_{\mathrm{LL}_{r}} .
\end{gathered}
$$

The electric utility needs to pay GHG emission penalties due to no-load losses in the case where $\Delta P_{\mathrm{NLL}}>0$. The same rule applies for the $\Delta P_{\mathrm{LL}}$. When this is not the case, the proposed environment cost can be thought as a benefit.

4.2. Environmental Cost Coefficient. Equation (8) presents the proposed loss evaluation technique by incorporating the environmental cost $(\mathrm{EC}-€)$. The environmental cost throughout the transmission line lifetime results from the total energy losses-no-load losses and load losses,

$$
\mathrm{EC}=\mathrm{EC}_{\mathrm{NLL}}+\mathrm{EC}_{\mathrm{LL}}
$$

The no-load losses part of transmission lines will not be encountered in our study due to being insignificant in comparison to load losses. Thus, the environmental cost is solely due to load losses, given by the following:

$$
\mathrm{EC}=B_{e} \times \Delta P_{\mathrm{LL}},
$$

where $B_{e}$ is the load loss environmental factor $(€ / \mathrm{kW})$ and $\Delta P_{\mathrm{LL}}$ is the rated load loss difference between an evaluated transmission line and a reference transmission line $(\mathrm{kW})$. The environmental factor $B_{e}$ is given by the following formula:

$$
B_{e}=\frac{\mathrm{LECL}_{e} \times \mathrm{TLF}^{2}}{\mathrm{ET} \times \mathrm{FCR} \times \mathrm{IF}},
$$

where $\mathrm{LECL}_{e}$ is the levelised annual environmental cost of load losses $(€ / \mathrm{kW}-\mathrm{yr})$ that are computed as follows:

$$
\begin{aligned}
\mathrm{LECL}_{e}= & \mathrm{CRF} \times \mathrm{HPY} \times C \times\left(\frac{1+\mathrm{EIR}_{e}}{d-\mathrm{EIR}_{e}}\right) \\
& \times\left[1-\left(\frac{1+\mathrm{EIR}_{e}}{1+d}\right)^{\mathrm{BL}}\right],
\end{aligned}
$$

where $C$ is the current year GHG emission cost factor $\left(€ / \mathrm{kWh}\right.$ ) explained in the following section and $\mathrm{EIR}_{e}$ is the annual escalation rate of the current year GHG emission cost.
TABLE 1: Transmission lines. Installation cost specifications.

\begin{tabular}{lccc}
\hline Line model & Cost $(€ / \mathrm{km})$ & Length $(\mathrm{km})$ & $\begin{array}{c}\text { Total cost } \\
\text { including } 4 \% \\
\text { admin. fees }\end{array}$ \\
\hline Type A $^{*}$ & $158,951.2$ & 45 & $7,438,915.8$ \\
Type B $^{*}$ & $194,336.3$ & 45 & $9,094,940.1$ \\
\hline
\end{tabular}

${ }^{*}$ These refer to real commercially available line conductors; however, their true names are not disclosed in this paper.

4.3. Current Year GHG Emission Cost Factor. The current year greenhouse gases emission cost $(C)$ used in formula (14) is evaluated as follows:

$$
C=C_{\mathrm{CY}} \times \sum_{I=1}^{N} f_{I} \times e_{I} .
$$

Equation (15) applies for all fuel types, fuels $I$ to $N$, used in the electricity generation mix. The $C_{\mathrm{CY}}$ factor is the current year GHG emission cost value in $\left(€ / t_{\mathrm{CO}_{2}}{ }^{-}{ }_{\mathrm{CO}_{2}}\right.$ are the annual equivalent tonnes of $\mathrm{CO}_{2}$ emissions), $f_{I}$ is the fraction of the delivered electricity coming from fuel $I$ and $e_{I}$ is the emission factor $\left(t_{\mathrm{CO}_{2}} / \mathrm{kWh}\right)$ for fuel type $I$ given by [10]

$$
\begin{aligned}
e_{I}= & \left(e_{\mathrm{CO}_{2}, I}+21 \times e_{\mathrm{CH}_{4}, I}+310 \times e_{\mathrm{N}_{2} \mathrm{O}, I}\right) \\
& \times \frac{0.0036}{n_{I} \times\left(1-\lambda_{I}\right)} .
\end{aligned}
$$

For this study three greenhouse gases are accounted for: carbon dioxide $\left(\mathrm{CO}_{2}\right)$, methane $\left(\mathrm{CH}_{4}\right)$, and nitrous oxide $\left(\mathrm{N}_{2} \mathrm{O}\right)$. The goal is to enumerate the equivalent emissions corresponding to each $\mathrm{kWh}$ of the produced energy and calculate their environmental cost. According to the type of the fossil fuel to be burned, each gas has a different global warming potential. Formula (16) converts all the emitted gases into equivalent $\mathrm{CO}_{2}$ emissions. $\mathrm{CH}_{4}$ is thus a 21 times more powerful gas than $\mathrm{CO}_{2}$ and $\mathrm{N}_{2} \mathrm{O}$ is a 310 times more powerful gas than $\mathrm{CO}_{2}$ [10].

In (16) the factors $e_{\mathrm{CO}_{2}, I}, e_{\mathrm{CH}_{4}, I}$, and $e_{\mathrm{N}_{2} \mathrm{O}, I}$ are the emission factors of $\mathrm{CO}_{2}, \mathrm{CH}_{4}$, and $\mathrm{N}_{2} \mathrm{O}$ for each fuel type in $\mathrm{kg} / \mathrm{GJ}$ [10]. The 0.0036 component is used so as to convert the $\mathrm{kg} / \mathrm{GJ}$ to $t_{\mathrm{CO}_{2}} / \mathrm{kWh}$. The $n_{I}$ component is the fuel conversion efficiency for each fuel type and the $\lambda_{I}$ represents the fraction of electricity lost in generation and transmission [7].

Equation (16) gives the flexibility to calculate a different environmental component according to the fuel type used in the electricity generation mix. Each fuel type has its own $\mathrm{CO}_{2}$ environmental component.

\section{Results and Discussion}

The proposed methodology (TOCE) as well as the conventional loss evaluation method (TOC) is used for the economic evaluation of transmission lines that are to be installed in the Cyprus Power System. Real financial data and system characteristics are utilized.

Two different transmission line models were evaluated the Type A and Type B. An indicative cost specification of the two models is shown in Table 1 [14]. The total purchase and 
installation price (PP) for Type A line is $€ 7,438,915$.8 and for Type B line is $€ 9,094,940.1$.

The loading profile of the models is assumed to be identical to the profile, under smooth operating conditions, of the Cyprus Power System. More precisely, unpredictable and unstable operating conditions are not considered. The losses evaluations runs are simulated for the years 2010-2020 during which sufficient information and predictions are available by the Electricity Authority of Cyprus (EAC). According to the electricity demand and the voltage level of the transmission lines, EAC has simulated forecasted load losses of the two lines for 2012 to 2020; loading losses for years 2010-2011 are already known (Table 2) [14]. For this paper the EAC operating profile and predictions are thus utilized.

The evaluated comparison made is between the conductors that operate at identical voltage level. There should be no comparison between conductors operating under different voltage ratings. This is due to the fact that transmission line conductors that operate at different voltage levels have different reference energy losses. Thus, Type A $132 \mathrm{kV}$ line is compared to Type B $132 \mathrm{kV}$ line, whereas Type A $220 \mathrm{kV}$ is compared to Type B $220 \mathrm{kV}$.

5.1. TOC Evaluation. The TOC of the two transmission line models is calculated using the aforementioned methodology [3]. The results are based on formulas (2)-(7). The $B$-factor is calculated according to the characteristics of the system, giving $B=511.2 € / \mathrm{kW}$. The total installation price for the models is shown in Table 1. The TOC prices (installation cost + lifetime losses cost (1)) for the lines for the different voltage levels are shown in Table 3.

The most cost-effective and "energy-efficient" transmission line is the one that has the lower TOC over the predetermined evaluation period at an exact voltage level. For this short-term loss evaluation study, the Type A model is found to be the finest choice of conductor for both voltage level operations. The Type B line costs 5.3\% more than the Type A line at $132 \mathrm{kV}$ and $14.8 \%$ more at $200 \mathrm{kV}$ voltage level.

5.2. TOCE Evaluation. The proposed TOCE that incorporates the environmental cost factor is evaluated in this section. From (8) the environmental cost (EC) needs to be calculated only, and then to be added to the conventional TOC The equations used are given in (5)-(16).

The current year $\mathrm{CO}_{2}$ emission factor (16) and its cost (15) were calculated using the standard emission factors for diesel combustion $[9,10]$ in the utility mix. The environmental load loss factor is $B_{e}=24.03 € / \mathrm{kW}$. The reference transmission line load loss used is $800 \mathrm{~kW}$ for both levels since the evaluation comparison that should be made is between identical voltage ratings. The TOCE prices (installation cost + lifetime losses cost with environmental cost (8)) for the lines under the different voltage ratings are shown in Table 4.

The most cost-effective and "energy-efficient" transmission line is the one that has the lower TOCE over the specified evaluation period. As applied with the conventional loss evaluation method, the Type A line was found to be the most cost-effective choice. In this case, Type B costs $3.98 \%$ more than Type A line at $132 \mathrm{kV}$ and $14.3 \%$ more at $220 \mathrm{kV}$.
TABLE 2: Transmission line load losses.

\begin{tabular}{ccccc}
\hline Year & $\begin{array}{c}\text { Type A } \\
132 \mathrm{kV}(\mathrm{kW})\end{array}$ & $\begin{array}{c}\text { Type B } \\
132 \mathrm{kV}(\mathrm{kW})\end{array}$ & $\begin{array}{c}\text { Type A } \\
220 \mathrm{kV}(\mathrm{kW})\end{array}$ & $\begin{array}{c}\text { Type B } \\
220 \mathrm{kV}(\mathrm{kW})\end{array}$ \\
\hline 2010 & 1990 & 1420 & 600 & 370 \\
2011 & 2170 & 1550 & 670 & 430 \\
2012 & 2360 & 1690 & 740 & 490 \\
2013 & 2580 & 1840 & 810 & 550 \\
2014 & 2810 & 2000 & 890 & 610 \\
2015 & 3060 & 2180 & 970 & 680 \\
2016 & 3340 & 2380 & 1070 & 750 \\
2017 & 3640 & 2600 & 1170 & 820 \\
2018 & 3940 & 2820 & 1270 & 900 \\
2019 & 4240 & 3040 & 1390 & 980 \\
2020 & 4540 & 3260 & 1510 & 1070 \\
\hline
\end{tabular}

TABLE 3: Transmission line TOC.

\begin{tabular}{ccccc}
\hline & Type A & Type B & Type A & Type B \\
& $132 \mathrm{kV}(€)$ & $132 \mathrm{kV}(€)$ & $220 \mathrm{kV}(€)$ & $220 \mathrm{kV}(€)$ \\
\hline TOC & $11,171,205.8$ & $11,761,280.1$ & $8,624,015.8$ & $9,903,807.1$ \\
\hline
\end{tabular}

TABLE 4: Transmission line TOCE.

\begin{tabular}{ccccc}
\hline Type A & Type B & Type A & Type B \\
& $132 \mathrm{kV}(€)$ & $132 \mathrm{kV}(€)$ & $220 \mathrm{kV}(€)$ & $220 \mathrm{kV}(€)$ \\
\hline TOCE & $11,504,745.8$ & $11,963,510.1$ & $8,643,735.8$ & $9,877,170.1$ \\
\hline
\end{tabular}

TABLE 5: Calculation of TOC/TOCE.

\begin{tabular}{lccc}
\hline Type A & Type B & Type A & Type B \\
$132 \mathrm{kV}$ & $132 \mathrm{kV}$ & $220 \mathrm{kV}$ & $220 \mathrm{kV}$ \\
\hline $97.1 \%$ & $98.3 \%$ & $99.7 \%$ & $100.27 \%$ \\
\hline
\end{tabular}

5.3. TOC and TOCE Benchmark. The numerical results of the conventional TOC and the proposed TOCE can be found in Tables 3 and 4 . The difference in the cost figures between the evaluation endeavors is the environmental cost of each line. Both evaluation methods have proved that the Type A transmission line is the most economically efficient. When the environmental factor is taken into account the cost is increased or decreased, according to the reference unit utilized.

For the Type A line at $220 \mathrm{kV}$ the cost is increased by $0.3 \%$ in respect to the cost calculated by the conventional method. For the Type A conductor operating at $132 \mathrm{kV}$ the increase in total owning cost is $2.9 \%$, and for the Type B line at $132 \mathrm{kV}$ the increase is $1.7 \%$. In contrast, the total owning cost of the Type B transmission line at $220 \mathrm{kV}$ is decreased by $0.27 \%$. Table 5 shows the contribution of the environmental cost, by calculating the ratio of the conventional total owning cost over the total owning cost incorporating environmental component (TOC/TOCE).

The reference unit losses can be seen as the limit for the "free emission allowance." In our study the "free emission allowance" limit is set constant at $800 \mathrm{~kW}$ for both methodologies. It is used as an arbitrary figure that allows the concept 
of "reference unit" to be understood. This is an empirical estimation that will need to be investigated further.

The Type B transmission line, operated at $220 \mathrm{kV}$, has a decreased value of annual load losses when incorporating the environmental cost. As given by (10), the difference term is negative: lower line load losses than the losses of the reference transmission line. In this case, the line is basically rewarded for the low losses that do not force the GHG emission limit to be violated. It can be seen as a source of financial saving for a utility; the losses cost to utility is thus decreased.

The contribution of the environmental factor and the "reference unit" is clearly visible by the results obtained using both methodologies. A vital part in the proposed methodology is the correct estimation of the reference losses and of the environmental cost for a utility.

\section{Conclusion}

This paper aims to introduce an emission trading scheme on the conventional transmission line loss evaluation method. The proposed technique is used for the economic evaluation of transmission lines embedded in the Cyprus Power System and the results are compared to the conventional total owning cost methodology (TOC). True financial data and system characteristics are utilized. A vibrant part in the proposed methodology is the correct estimation of the reference losses and of the environmental cost for a utility. Future work will be carried out towards accurately establishing the reference transmission unit loss specifications and permissible GHG emissions credits.

\section{References}

[1] R. Targosz, The Potential for Global Energy Savings From High Efficiency Distribution Transformers, Leonardo Energy, 2005.

[2] CERA, "A strategic plan for the promotion of renewable energy sources in the Cyprus electricity generation system," Tech. Rep., 2010.

[3] IEEE standard C57.120-1991, IEEE Loss Evaluation Guide For Power Transformers and Reactors, 1991.

[4] D. L. Nickel and H. R. Braunstein, "Distribution transformer loss evaluation: I-proposed techniques," IEEE Transactions on Power Apparatus and Systems, vol. 100, no. 2, pp. 788-797, 1981.

[5] D. L. Nickel and H. R. Braunstein, "Distribution transformer loss evaluation: I-load characteristics and system cost parameters," IEEE Transactions on Power Apparatus and Systems, vol. 100, no. 2, pp. 798-811, 1981.

[6] D. G. Boice, R. J. Gursky, and J. B. Trad, "Cost of electrical power system losses for use in economic evaluations," IEEE Transactions on Power Systems, vol. 4, no. 2, pp. 586-593, 1989.

[7] P. S. Georgilakis and E. I. Amoiralis, "Distribution transformer cost evaluation methodology incorporating environmental cost," IET Generation, Transmission and Distribution, vol. 4, no. 7, pp. 861-872, 2010.

[8] G. Daskalakis, D. Psychoyios, and R. N. Markellos, "Modeling $\mathrm{CO}_{2}$ emission allowance prices and derivatives: evidence from the European trading scheme," Journal of Banking and Finance, vol. 33, no. 7, pp. 1230-1241, 2009.

[9] F. Zabihian and A. Fung, "Fuel and GHG reduction potentials by fuel switching and technology improvement in the Iranian electricity generation sector," International Journal of Engineering, vol. 3, no. 2, pp. 159-173, 2009.

[10] H. S. Eggleston, L. Buendia, K. Miwa, T. Ngara, and K. Tanabe, "IPCC Guidelines for National Greenhouse Gas Inventories," in The Intergovernmental Panel of Climate Change (IPCC '06), vol. 2 of Energy, 2006.

[11] P. S. Georgilakis, J. C. Olivares, R. Escalera-Perez, I. Fofana, and G. K. Stefopoulos, "Environmental cost of transformer losses for industrial and commercial users of transformers," in Proceedings of the North American Power Symposium, August 2011.

[12] P. S. Georgilakis, "Environmental cost of distribution transformer losses," Applied Energy, vol. 88, no. 9, pp. 3146-3155, 2011.

[13] E. I. Amoiralis, P. S. Georgilakis, M. A. Tsili, and A. T. Souflaris, "Utility-based economic assessment of distribution transformers considering specific load characteristics and environmental factors," Journal of Optoelectronics and Advanced Materials, vol. 10, no. 5, pp. 1184-1191, 2008.

[14] Electricity Authority of Cyprus, "Connection of "Vasilikos" power station with transmission Substation of "Tseri" 220/ 132KV," Tech. Rep., Transmission/Distribution Department of E.A.C., 2003. 

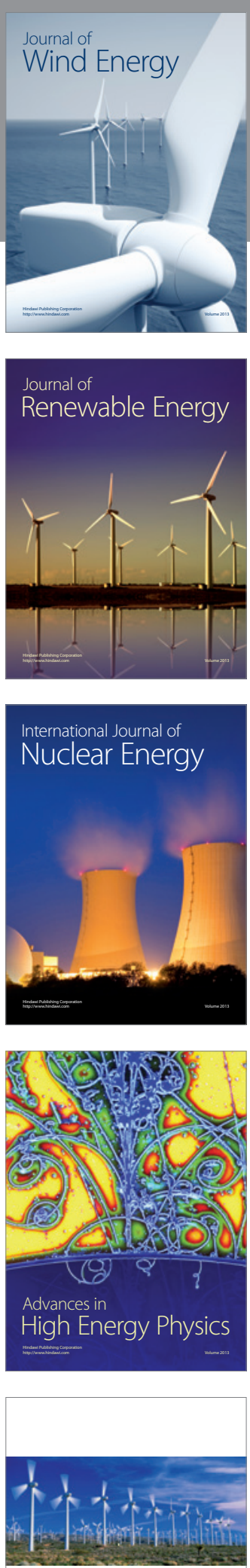

ISRN

Renewable Energy
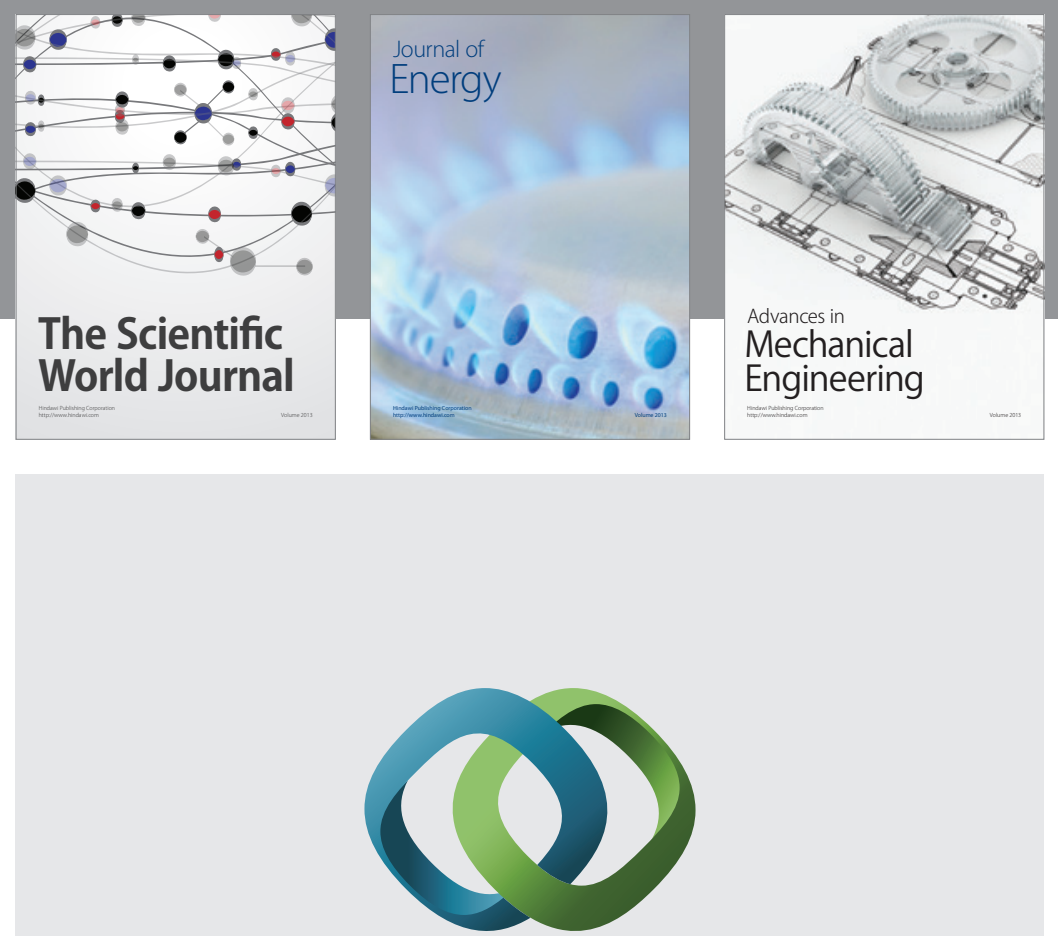

\section{Hindawi}

Submit your manuscripts at http://www.hindawi.com
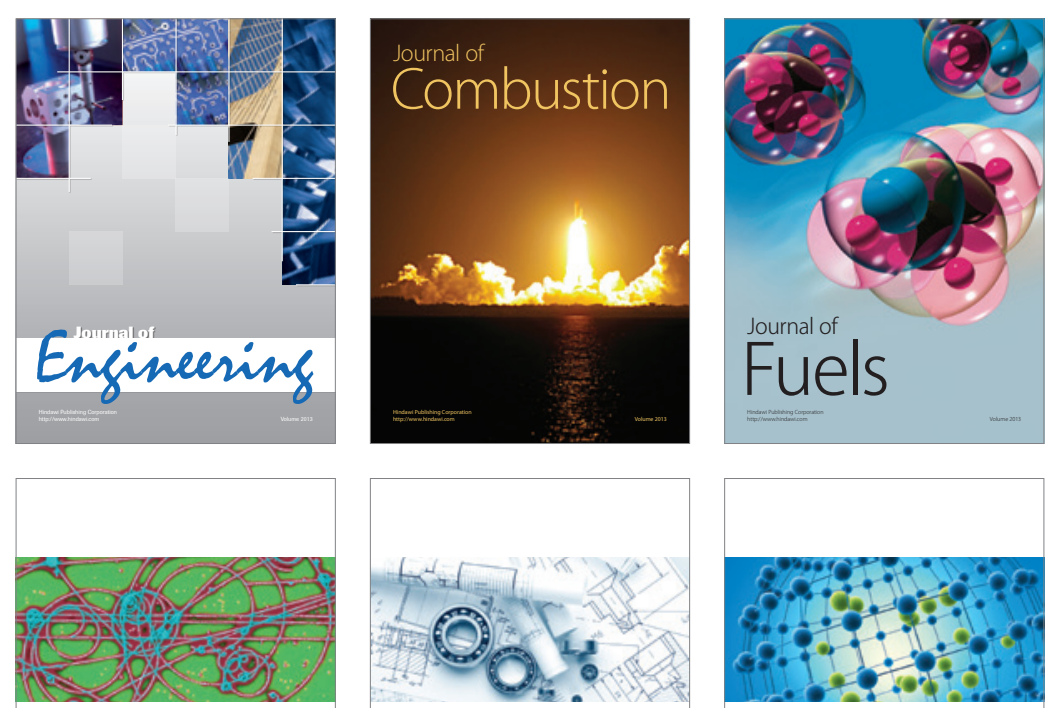

ISRN

High Energy Physics

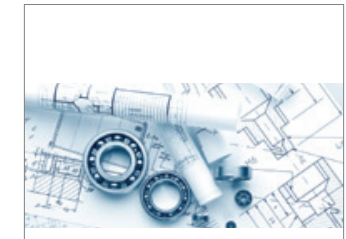

ISRN

Mechanical

Engineering

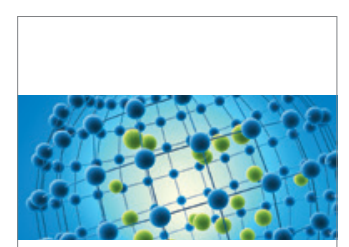

ISRN

Chemical

Engineering
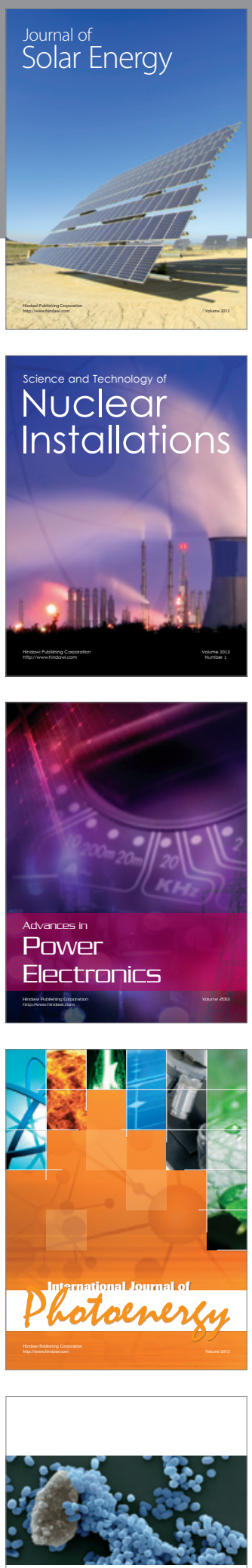

ISRN

Biotechnology 

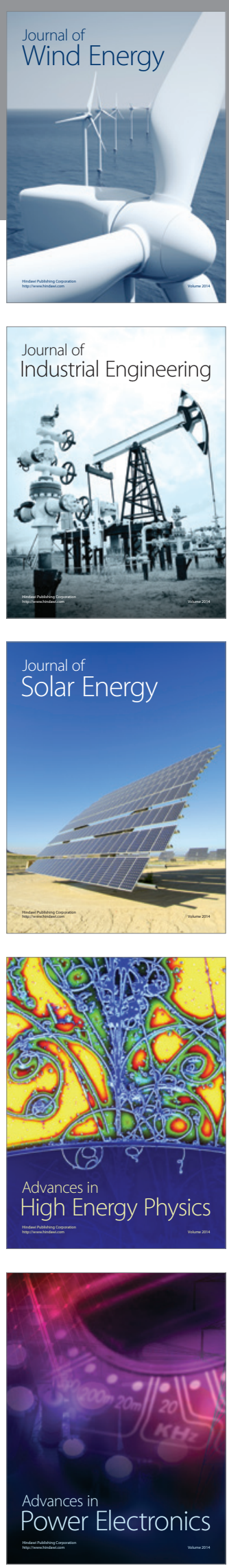
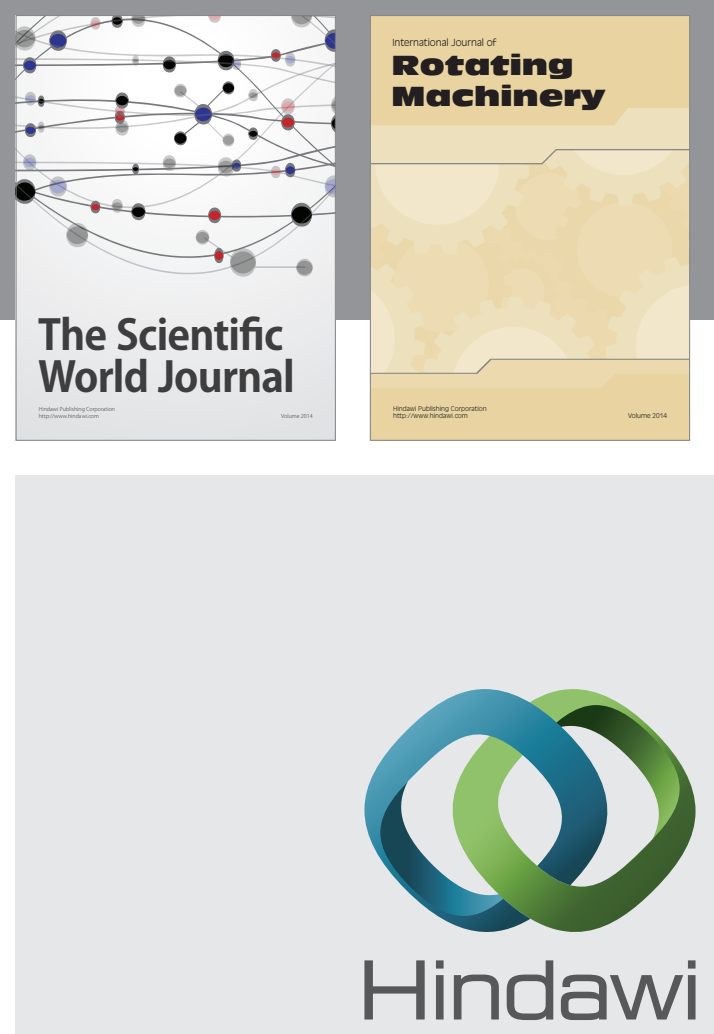

Submit your manuscripts at

http://www.hindawi.com
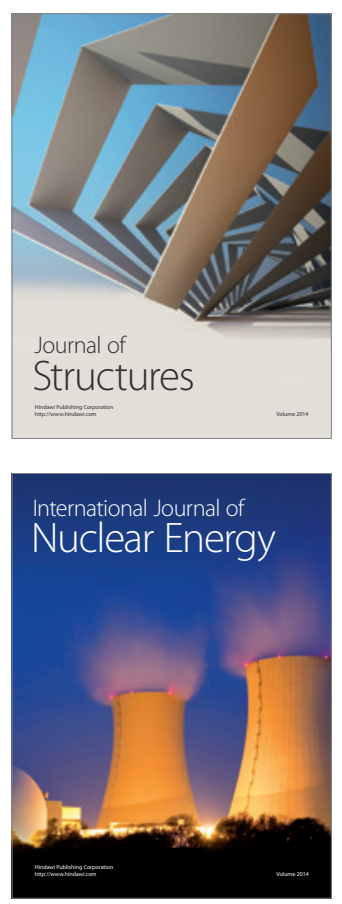
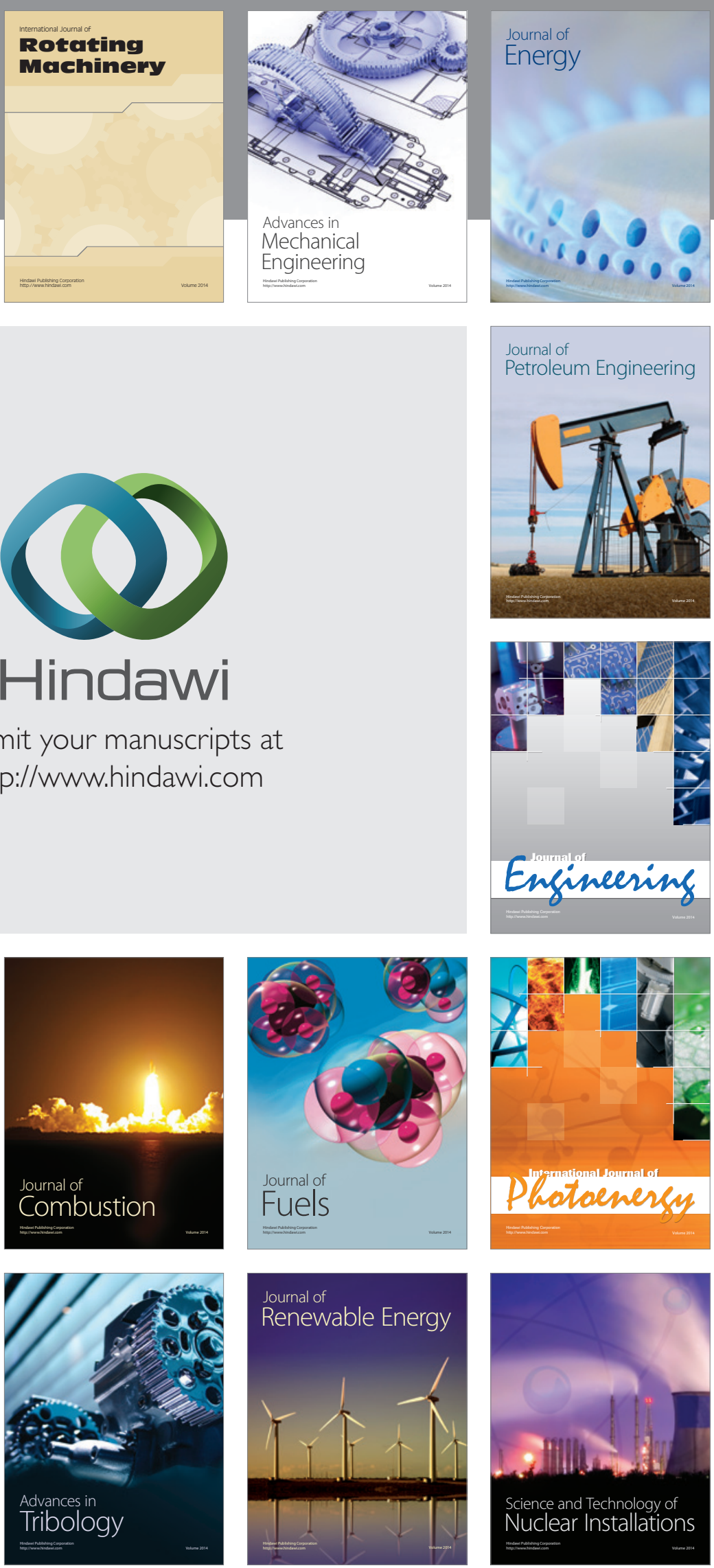